\title{
Polysèmes
}

Revue d'études intertextuelles et intermédiales

\section{Couleur des mots, éloquence de la couleur : Art Work de A.S. Byatt, ou l'écriture du silence}

\section{Laurence Petit}

\section{(2) OpenEdition}

\section{Journals}

Édition électronique

URL : http://journals.openedition.org/polysemes/627

DOI : $10.4000 /$ polysemes.627

ISSN : 2496-4212

Éditeur

SAIT

Édition imprimée

Date de publication : 1 janvier 2008

Pagination : 43-57

ISSN : 0999-4203

\section{Référence électronique}

Laurence Petit, «Couleur des mots, éloquence de la couleur : Art Work de A.S. Byatt, ou l'écriture du silence », Polysèmes [En ligne], 10 | 2008, mis en ligne le 01 mars 2015, consulté le 01 mai 2019. URL : http://journals.openedition.org/polysemes/627 ; DOI : 10.4000/polysemes.627

Ce document a été généré automatiquement le 1 mai 2019.

Polysèmes 


\title{
Couleur des mots, éloquence de la couleur : Art Work de A.S. Byatt, ou l'écriture du silence
}

\author{
Laurence Petit
}

1 La fascination qu'éprouve la romancière anglaise A.S. Byatt pour les formes d'expression à la fois verbales et visuelles s'exprime par un type de fiction qui repense la distinction traditionnelle entre les arts pour essayer de réussir ce qui semble être de prime abord une tâche impossible, lire un texte visuellement, ou visualiser un tableau textuellement. Cette « mise en tableau » de textes s'opère souvent par la " mise en texte » ekphrastique de tableaux existant réellement. Son recueil de nouvelles intitulé The Matisse Stories ${ }^{1}$, publié en 1993 et fondé sur trois tableaux du grand maître du modernisme, est précisément un exemple de cette mise en scène constante et simultanée du texte et de l'image dans son œuvre, par laquelle, comme le suggère Catherine Mari, «The Matisse Stories se donnent à lire mais aussi à voir $»^{2}$. En raison des contraintes formelles de la nouvelle comme genre, à savoir compression du sens et brièveté de la forme, ainsi que de sa capacité à saisir un moment de perception "unique », pour reprendre l'expression d'Edgar Allan Poe ${ }^{3}$, la nouvelle a souvent été comparée aux arts visuels, et en particulier à la peinture et à la photographie ${ }^{4}$. Les nouvelles qui vont encore plus loin en se fondant sur des tableaux ou des photographies existant réellement accentuent ainsi ce phénomène et se prêtent à toutes sortes d'expérimentations esthétiques en intermédialité visuelle et verbale en adoptant des stratégies narratives hors du commun qui franchissent les frontières du littéraire pour imiter le pictural.

2 La nouvelle "Art Work» illustre ce processus en se présentant comme l'équivalent textuel des expérimentations visuelles de Matisse en matière de couleur et de composition. Bien que Matisse soit avant tout considéré comme le chantre de la couleur, il y a dans son travail un lien très étroit entre couleur et composition, la juxtaposition des couleurs étant précisément ce qui donne sa forme au tableau. La couleur devient ainsi «la représentation signifiante de la forme », selon Matisse, suggérant par là la capacité qu'a la couleur à exprimer du sens et par conséquent à acquérir le statut et la fonction d'un 
véritable langage. Raoul Dufy, un contemporain de Matisse, affirme même que les couleurs sont «les mots du langage de la peinture». Dans son ouvrage critique récent Portraits in Fiction ${ }^{5}$, dans lequel elle étudie la fonction des portraits dans les œuvres littéraires, y compris les siennes, Byatt défend une thèse semblable, quoiqu'inversée : si les couleurs peuvent rendre le langage de la peinture, le langage devrait de la même manière être capable de rendre les couleurs de la peinture. Mais comment peut-on matériellement transcrire la couleur en mots autrement que par le biais de la simple référentialité ? Et si, dans les tableaux de Matisse, la couleur est bel et bien forme, comment la transcription, ou plutôt l'inscription de la couleur dans le texte peut-elle devenir à son tour le principe compositionnel de ce texte-même? Dans la nouvelle " Art Work », qui se présente comme un vibrant hommage au grand maître décédé, Byatt semble relever le défi et aller à l'encontre du poète Louis Aragon, qui déclarait dans son livre Matisse-en-France : «J'ai beaucoup écrit de Matisse sans jamais prétendre à rendre avec les mots la forme ni la couleur de ses tableaux $»^{6}$. À l'inverse, Byatt semble précisément s'efforcer de rendre avec des mots la forme et la couleur des tableaux de Matisse, et d'un en particulier, Le Silence habité des maisons, dont l'ekphrasis (c'est-à-dire la représentation verbale d'une représentation visuelle) ${ }^{7}$ ouvre le récit et génère l'histoire.

3 Afin de montrer comment ces deux notions fondamentales de couleur et de composition « in-forment » au sens littéral et figuré à la fois l'œuvre de Byatt et celle de Matisse, je me propose d'examiner les stratégies narratives déployées dans le texte, en particulier à travers un personnage central, Mrs Brown, personnage « haut en couleur " s'il en est. Si la couleur fonctionne et pour Matisse et pour Byatt comme principe de composition dans leur œuvre, dans la fiction de Byatt, ce n'est pas la juxtaposition des couleurs, si essentielle chez Matisse, qui fournit la forme, mais le tissage ténu de la couleur dans la trame ou toile même du texte. Byatt parvient à tisser la couleur dans le texte à travers le portrait du personnage féminin central de Mrs Brown, la femme de ménage mulâtre d'une famille d'artistes aisée comprenant le peintre Robin, sa femme Debbie, rédactrice en chef du magazine de design A Woman's Place, et leurs deux enfants, Natasha et Jamie. À travers la fonction de ce personnage dans le texte et ses interactions avec les autres protagonistes de l'histoire - d'abord en tant que femme de ménage pendant dix ans puis en tant qu'artiste au talent reconnu et stimulant à la fin de l'histoire -, se dessine un récit verbal dont l'esthétique rappelle celle du grand maître de la couleur et de la forme Matisse, à la fois dans son traitement de la couleur et dans sa conception de la forme. Cependant, Byatt va au-delà de l'expérimentation interartistique pour ré-examiner textuellement ces deux concepts picturaux en des termes qui rappellent les notions postmodernes de réseau et d'hybridité. Plus qu'un simple hommage à Matisse et à la couleur, la nouvelle apparaît alors comme un hommage à l'hétérogénéité postmoderne, dans lequel « l'œuvre d'art » du titre est elle-même un hommage au passé et au présent, à l'histoire et à la mythologie, à l'Orient et à l'Occident, au surnaturel et au quotidien, et bien sûr, comme toujours chez Byatt, à la visualité et à la textualité. La métaphore centrale de la toile, tantôt toile d'araignée au sens littéral, tantôt toile d'araignée au sens métaphorique, devient ainsi le nouveau principe compositionnel d'une trame textuelle et visuelle dans laquelle la couleur, comme chez Matisse, reste essentielle, mais au lieu d'être juxtaposée afin de réaliser cette « synthèse géométrique » de l'œuvre qui lui est si chère, est tissée au sein même du texte verbal et visuel. Mrs Brown, la figure de l'artiste dans le texte, et Byatt l'écrivain apparaissent toutes deux comme tissant le sort des personnages dans la toile de leur œuvre d'art respective, tout en présidant à leurs destinées. Toutefois, ce qui se dégage de cette expérimentation en visualité textuelle ou 
en textualité visuelle à travers l'utilisation de la couleur est en fait une réflexion sur les limites à la fois du langage et de la représentation. Au-delà de son hommage à Matisse et à sa théorie de la couleur, la nouvelle peut se lire paradoxalement comme un hommage en mots au silence prégnant à la fois de la couleur et des images et comme un questionnement radical de la notion même de représentation.

Comme dans les autres nouvelles des Matisse Stories, Byatt dans "Art Work» semble rendre hommage à Matisse, le chef de file de cette intense célébration de la couleur que fut le fauvisme, en essayant d'exprimer verbalement non seulement l'esprit mais aussi le style de son œuvre. Comme les deux autres nouvelles du recueil, «Art Work » est fondé sur l'intertexte pictural d'un tableau de Matisse, dans le cas présent Le Silence habité des maisons ${ }^{8}$, précédé d'un épigraphe pictural sous la forme d'un dessin de Matisse, ici L'Artiste et le modèle reflétés dans le miroir 9 . En plus de ces références interpicturales évidentes, le texte comporte aussi de nombreuses références implicites ou explicites à l'univers esthétique du peintre, que je n'énumérerai pas ici. Pourtant, au-delà de ces nombreuses références à Matisse qui parsèment le texte, c'est précisément l'intertexte pictural central de la nouvelle, Le Silence habité des maisons, qui gouverne la stratégie narrative centrale et permet à Byatt de célébrer le peintre tout en abordant des notions picturales formelles telles que la couleur et la composition. Byatt présente en effet le texte de "Art Work " d'emblée comme une tentative de restauration de la couleur et des mots de l'image visuelle inaugurale, en l'occurrence une reproduction en noir et blanc (tirée du livre intitulé Matisse de Sir Lawrence Gowing ${ }^{10}$ ) du Silence habité des maisons, dont l'ekphrasis constitue l'ouverture de la nouvelle. Tout en déplorant l'absence de couleurs reproduites hormis quelques vagues teintes de gris, la voix narrative s'empresse de noter à quel point il est tentant de rendre au tableau ses couleurs d'origine manquantes grâce aux pouvoirs créateurs de l'imagination. Le discours ekphrastique de l'historien ou du critique d'art par lequel s'ouvrait le récit fait très vite place à une rêverie mentale dans laquelle le narrateur cède à une impulsion coloriste, restituant du même coup au regard du lecteur les couleurs d'origines du tableau telles qu'il les imagine. Tout fonctionne comme si la reproduction en noir et blanc, dont l'obscurité est constamment soulignée, se révélait illisible précisément du fait de cette absence de couleur, comme si la couleur était à la fois la source et la condition de la production de sens dans le tableau. Le truchement des mots est ainsi utilisé pour compléter le processus imaginaire afin de rétablir les couleurs de la reproduction en noir et blanc. Dans le même temps, en soulignant deux fois la présence incongrue dans le tableau d'un " très grand livre blanc » ( $a$ « huge white book », 31), dont un enfant tourne les pages, la voix narrative semble suggérer que ces pages blanches sont là pour inviter le spectateur à les remplir de mots, tout comme ces ovales "vides » («blank », 31) et « dénués de traits» («featureless», 31) figurant les visages des deux personnages du tableau qui semblent attendre qu'on achève de les dessiner et de les colorier. Dans les pages suivantes de la nouvelle, après une phrase de transition subtile qui rappelle le titre même du tableau ("There is an inhabited silence in 49 Alma Road », 32) et qui marque le glissement de l'image au texte ainsi que le début de l'histoire effective, le lecteur est plongé à la fois dans l'univers du tableau et dans celui de la fiction par un narrateur qui, effectivement, dessine et colorie les " contours tracés à la craie » («the chalked outline», 31) des personnages du tableau et leur invente des vies imaginaires par-delà la surface plane de l'image. C'est un peu comme si la voix narrative, par le biais des mots, se fixait une double tâche, celle de convertir l'image d'une image (en l'occurrence la reproduction en noir et blanc) à la fois en une image plus adéquate, en lui restituant les couleurs originelles de la matrice, et en un texte, en remplissant les pages 
blanches du livre dans le tableau avec l'histoire fantasmée de ce même tableau. Ce livre " peint » devient ainsi le locus d'une intéressante et double mise en abyme, dans laquelle les pages tournées par l'enfant contiennent potentiellement la transcription en mots du monde imaginaire qui les entoure et sont, dans le même temps, les pages mêmes de la nouvelle «Art Work » que nous nous apprêtons à lire. Par conséquent, de même que le lecteur de la nouvelle "Art Work» de Byatt est transporté d'emblée dans l'univers pictural du Silence habité des maisons de Matisse, le spectateur de ce tableau, tel qu'il se matérialise à travers l'ekphrasis de sa reproduction en noir et blanc (ou bien apparaitt dans une autre reproduction, en couleur cette fois, sur la couverture de l'édition Random House du recueil) est transporté dans l'univers textuel de la fiction de Byatt. Il y a donc une mise en scène constante et simultanée du texte et de l'image par laquelle le texte de Byatt est « mis en tableau », pour reprendre l'expression de Catherine Mari ${ }^{11}$, et le tableau de Matisse « mis en texte».

Cette célébration textuelle de Matisse est avant tout une célébration du miracle de la couleur dans un univers précisément saturé de couleurs, allant des personnages bigarrés et des tons criards des programmes de télévision et des magazines de design à l'élégance chromatique soigneusement étudiée de la galeriste Shona McRury, véritable œuvre d'art ambulante. Cette célébration de la couleur, c'est-à-dire de toutes les couleurs, devient encore plus évidente dès que le lecteur rencontre la femme de ménage Mrs Brown, le personnage central de la nouvelle. Avec ses ensembles en patchwork extravagants et ses turbans fleuris aux couleurs de l'arc-en-ciel, Mrs Brown semble tout droit sortie d'un tableau de Matisse, synthèse de la Femme au chapeau lumineuse et irisée de $1905^{12}$, de la séries des Chapeaux à plumes de $1919^{13}$ représentant son modèle Antoinette portant des chapeaux extraordinairement inventifs et décoratifs, et de sa conception de la "femmeplatane ", ou "femme-bouquet ", sorte de Nouvelle Eve sensuelle et florale poussant dans l'Éden reconstitué que figure l'atelier du peintre ${ }^{14}$. Cependant, ce n'est pas seulement grâce à ces tenues exotiques et irisées que Mrs Brown s'impose comme le vecteur idéal de la célébration de la couleur que nous offre Byatt dans cette nouvelle. Mrs Brown, en effet, ne se contente pas d'afficher à elle seule le spectre complet des couleurs sur ses vêtements et ses turbans : elle est Couleur, avec un « $C$ » majuscule, c'est-à-dire qu'elle est l'incarnation textuelle de ce concept pictural si central chez Matisse. Son nom même, Mrs Brown, l'associe ontologiquement, et pas seulement mimétiquement, avec la couleur brune, qui elle-même représente une synthèse des tons fauves, suggérant par là qu'elle n'est pas seulement une couleur quelconque, mais la Couleur matissienne par excellence. Par ailleurs, son corps même l'identifie avec la Couleur, non seulement à cause de la couleur de sa peau, «ni noire ni brune mais une sorte de jaune orangé » (« neither black nor brown but a kind of amber yellow », 39), due à ses origines mixtes, «mi-guyanaise, mi-irlandaise » ("part-Guyanese, part-Irish», 41), mais aussi à cause de ses nombreux bleus résultant des coups que ne cesse de lui infliger Hooker, le père de ses deux garçons. En ce sens, les effets chromatiques saisissants produits par ces bleus suggèrent que Mrs Brown, tout comme Shona McRury, est aussi à sa façon une œuvre d'art ambulante ainsi qu'une palette d'artiste constamment renouvelée. Sa fonction et son essence en tant que Couleur dans le texte s'expriment à travers la forme d'art originale qui la caractérise, et qui se manifeste d'abord par les tricots qu'elle offre aux enfants de la famille et par ses propres vêtements faits main, avant de culminer par une exposition à la toute fin de l'histoire dans laquelle elle vole la vedette («steals the show » au sens propre et figuré) à son employeur Robin, peintre de petite renommée. Tout comme ses vêtements extravagants, les productions artistiques de Mrs Brown en patchwork tricoté et brodé 
sont bigarrées et irisées, aux antipodes de l'utilisation de la couleur que fait le peintre Robin, soulignant par là une opposition fondamentale entre deux perspectives esthétiques de la couleur et de l'art qui, par de nombreux aspects, représentent les deux pôles des propres théories esthétiques de Matisse. Là où Robin représente le pôle intellectuel, cérébral, rationnel, spirituel, purifié, et dématérialisé de l'approche matissienne de la lumière et de la couleur, le «multicolorisme» ou "pancolorisme » païen de Mrs Brown incarne le pôle débridé, sensuel, ludique, impulsif, naturaliste, et anti-conformiste, celui de la «Joie de vivre » et de la « volupté sublimée » de Matisse.

Ce qui est particulièrement intéressant lorsque l'on considère Mrs Brown comme l'incarnation textuelle, voire l'inscription textuelle de la couleur dans cette nouvelle de Byatt, c'est que Mrs Brown ne représente pas seulement la Couleur dans le texte. En tant que Couleur, elle est aussi, comme dans les tableaux de Matisse, le principe compositionnel de l'œuvre, en d'autres termes le facteur d'harmonie, d'équilibre et d'unité qui soutient toutes les parties de l'ensemble. Comme nous l'avons vu précédemment, la couleur chez Matisse est aussi la forme, en ce sens que la couleur est ce qui fournit au tableau sa cohérence interne, et sous de nombreux aspects, Mrs Brown est précisément le personnage qui « in-forme » le texte au sens propre comme au sens figuré. Avant même de parler de l'impact durable qu'elle va avoir en «façonnant » les destinées de la famille Dennison et par là même en donnant au récit sa cohérence interne, il est utile de remarquer à quel point, en tant que femme de ménage, Mrs Brown représente le principe organisateur et de la maison et de ses occupants, en étant à la fois un facteur d'unité spatiale ou topologique à travers le maniement de son aspirateur, et un facteur d'unité morale et familiale par sa fiabilité professionelle, ou "professional reliability " (42-43), terme qui, en anglais, signifie étymologiquement "capacité à lier ou relier ». Mrs Brown est par conséquent non seulement celle qui veille littéralement à ce que l'espace de la maison soit en ordre, mais aussi métaphoriquement celle qui fait que tout tienne ensemble, y compris la vie de ses employeurs. Toutefois, si Mrs Brown en tant que personnification de la couleur est ainsi le principe compositionnel de la maison où elle travaille comme femme de ménage, réussissant là, tout comme Matisse dans ses tableaux, une sorte de "synthèse spatiale » ou "géométrique " ainsi qu'une synthèse « morale ", c'est dans sa forme d'art tout à fait particulière que ce souci d'harmonie, d'ordre, d'équilibre, de synthèse et d'unité trouve son paroxysme. Que ce soit dans les vêtements qu'elle confectionne ou dans la caverne extravagante qu'elle a construite pour son exposition à la fin de la nouvelle, Mrs Brown, dont l'expression artistique hybride et très postmoderne a pour forme dominante le «patchwork» (60-71-78), juxtapose et «fabrique » des couleurs, des tissus et toutes sortes de matériaux avec une inventivité et une énergie inégalées, culminant dans cette "Caverne d'Aladdin aux couleurs éclatantes » (" this brilliantly coloured Aladdin's Cave», 77) dans laquelle elle présente une allégorie des travaux ménagers contemporains pleine de dérision et d'auto-dérision. Et malgré l'extraordinaire hétérogénéité et l'apparent chaos de ce lieu insolite, ce qui ressort de la longue description qu'en fait le narrateur, ce sont les liens extrêmement étroits qui en unissent toutes les composantes, connectées par quelques "nœuds » structuraux et thématiques, avec, au cœur de cette allégorie, le dragon en forme d'aspirateur désormais vaincu, «inerte et suffocant» (80). Une étude plus poussée de l'univers esthétique de la caverne fait apparaitre tour à tour tous les occupants de la maison et protagonistes de l'histoire, révélant ainsi la structure extrêmement élaborée de l'ensemble, et le souci de composition ayant présidé à sa réalisation. Cette œuvre d'art forme un tout également grâce à trois éléments connecteurs, les « énormes tapisseries » 
(77) en tricot accrochées au mur, les « toiles d'araignées au crochet » (77) qui pendent du plafond, et le « fil qui court de compartiment en compartiment» (78) dans la commode. Ces éléments connecteurs font tous référence directement ou indirectement à l'activité de Mrs Brown comme femme de ménage ou à ses intérêts en matière artistique, qui ont tous, d'une manière ou d'une autre, partie liée au tissage, Mrs Brown étant d'ailleurs ellemême désignée par le narrateur comme "cette prolifique tisseuse de toiles éclatantes " ("that prolific weaver of bright webs", 90). En plus de la référence évidente à ses productions artistiques, il est tentant, vu l'impact qu'ont eu sur cette famille ses dix années passées à leur service en tant que femme de ménage, de la considérer non pas seulement comme une couturière postmoderne sortant de l'ordinaire et devenue soudain très médiatique, mais peut-être aussi comme une sorte de figure d'araignée tissant sa toile lentement mais sûrement tout au long de l'histoire et produisant à la fin une œuvre d'art "naturelle », bien qu'extrêmement élégante et raffinée. Cette image de l'araignée ainsi que la référence à un processus de tissage polymorphe que le texte mettrait en œuvre peut nous permettre de considérer Mrs Brown comme une sorte de figure mythologique double. On peut bien sûr l'interpréter comme l'une des Parques, tricotant non seulement les pulls et les écharpes de la famille Dennison, mais aussi leurs destinées, ou bien on peut voir en Mrs Brown une sorte d'Arachné mythologique, condamnée par Athéna à ne jamais cesser de tisser ${ }^{15}$. À travers la description de la caverne, qui fonctionne comme une représentation allégorique de la maison et de ses tâches ménagères, Mrs Brown apparaît comme s'étant « tissée » elle-même dans son œuvre d'art en tant que pourchasseuse d'araignées et araignée tout à la fois, " aspirant ", si l'on peut dire, à présider aux destinées de toute la famille telle une figure protectrice. À un niveau supérieur, c'est le texte lui-même qui, par son étymologie "texere ", " tisser ", peut être considéré également comme une gigantesque toile dans laquelle l'écrivain/araignée Byatt tisse le sort de ces personnages fictifs. Au sein de cette triple «toile» (toile du texte, toile/tableau, et toile d'araignée), c'est la Couleur elle-même qui est en fait tissée dans la trame du texte en tant que principe compositionnel garantissant sa structure et sa forme. On voit ainsi clairement se dessiner l'analogie entre la nouvelle « Art Work» de Byatt et les tableaux de Matisse en ce qu'ils utilisent tous deux la Couleur comme principe structurant, la différence étant que là où Matisse juxtapose les couleurs pour ses compositions, Byatt tisse la Couleur dans la «toile » de son texte tout en utilisant dans le même temps la Couleur même pour générer, ou « tisser », le texte.

7 Mais ce n'est pas là la seule manière dont Mrs Brown s'est inscrite dans cette œuvre d'art visuelle allégorique. En plus de figurer sa présence à la fois comme pourchasseuse d'araignées et comme araignée tissée dans la toile de l'œuvre tout autant que la tissant, Mrs Brown s'est aussi littéralement écrite, ou "inscrite ", dans la structure visuelle de son œuvre sous la forme d'une "série de lettres, dorées sur un beau fond couleur chocolat » (" a series of letters, gold on rich chocolate », 81), que quelqu'un est en train de suspendre sur « une sorte de fil à linge hi-tech à l'aide de minuscules pinces à linge rouge sombre " (« on a kind of hi-tech washing-line with tiny crimson pegs », 81). Ces lettres, qui forment les mots suivants «Sheba Brown dans différents tissus 1975-1990» (81), ne représentent pas seulement une forme de signature d'artiste autotélique amusante, étant donné sa carrière précédente comme femme de ménage. Elles révèlent aussi le choix délibéréré de Mrs Brown de se transformer elle-même en un texte, un objet-texte visuel et « concret » suspendu à un fil à linge comme n'importe quel vêtement. Là aussi, comme pour l'araignée et la pourchasseuse d'araignées, Mrs Brown est doublement présente dans l'œuvre, comme ce « quelqu'un » anonyme, vraisemblablement une femme de ménage, en 
train de suspendre les lettres/vêtements, et comme les lettres mêmes qui proclament son nom et par conséquent la représentent dans son identité. Par le biais de ces lettres, elle s'inscrit et se figure elle-même littéralement dans son œuvre dans une sorte «d'expressionnisme gestuel» typique de l'art contemporain selon Henry-Claude Cousseau ${ }^{16}$. Elle est aussi doublement présente en tant que Couleur, puisque, en tant que Couleur, elle est «inscrite» dans l'œuvre d'art, comme nous venons de le voir, et également parce que ces lettres qui la définissent sont elles-mêmes des lettres en couleur imitant les couleurs de sa peau, le «doré sur un beau fond couleur chocolat » rappelant une description antérieure du narrateur évoquant « les taches chocolat et violette sur sa peau dorée » (the " chocolate and violet stains on [her] gold skin », 44). Le récit introduit ainsi ces lettres comme étant essentiellement des couleurs, c'est-à-dire des signifiants, avant de représenter un texte pouvant être lu et déchiffré, en d'autres termes avant de véhiculer un sens ou un signifié. La dimension visuelle de ces lettres l'emporte par conséquent sur leur sens textuel, suggérant par là que Mrs Brown est bel et bien, avant tout autre chose, Couleur, et que c'est à travers cette reconnaissance de son identité en tant que couleur qu'elle peut exister à la fois comme Femme, et pas seulement comme " femme de ménage », et comme artiste. Cette consécration de Mrs Brown comme artiste à part entière à travers la présentation visuelle de son identité est présentée, malgré le processus de réification qu'elle implique, comme un véritable couronnement, celui de la «Reine» de "Saba» Brown ("Queen Sheba Brown»), dont le prénom majestueux est révélé ici pour la première fois dans la nouvelle, un couronnement souligné par la présence, sous les lettres, d'une photo la présentant «sous une sorte d'extravagante couronne formée par un entrelacs d'écharpes " ("under a kind of wild crown of woven scarves ", 81), ce qui, à son tour, renvoie à une description antérieure de la chevelure de Mrs Brown «[se dressant] comme la couronne du roi dans un jeu de cartes» (« [rising] like the crown of a playing-card king ", 39). Ce que cette glorification royale de Mrs Brown figure avant tout, c'est le couronnement de la couleur dans l'espace du texte, comme si Mrs Brown, en plus de rendre la couleur à Robin, qui désormais peint des formes « aux couleurs vives" "à la Mrs Brown » (89), avait aussi rendu la couleur aux mots eux-mêmes. En effet, nous constatons là non pas que la couleur est «sous l'obédience des mots ", comme ce fut le cas pendant des siècles, d'après Jean-Pierre Guillerm dans Des Mots et des couleurs ${ }^{17}$, avec des lettres qui se trouveraient être en couleur, mais que la couleur est elle-même littéralement transformée en texte avec Mrs Brown s'écrivant, ou plutôt s'inscrivant elle-même comme chair colorée dans son œuvre d'art.

8 Nous avions débuté cette lecture de la nouvelle «Art Work» de A.S. Byatt en montrant comment le narrateur, méditant sur la reproduction en noir et blanc du Silence habité des maisons de Matisse, utilisait des mots pour essayer de lui restituer ses couleurs manquantes, et comment, de la même manière, Byatt elle-même semblait essayer, dans son texte, de retrouver les couleurs du tableau d'origine. Cette quête des couleurs manquantes transforme l'écriture de Byatt en une véritable entreprise de restauration de la couleur non seulement à l'image par le truchement des mots mais aux mots mêmes par le truchement de l'image. Et pourtant, au-delà d'une simple tentative de « redonner des couleurs aux mots » et de "redonner des mots aux couleurs », Byatt semble ici rendre textuellement un tableau particulier de Matisse intitulé Le Silence habité des maisons, c'està-dire rendre textuellement le silence même, si l'on considère la peinture comme une forme d'expression non-linguistique, et par conséquent silencieuse. Mais comment rendre le silence, même si le silence évoqué dans l'hypallage du titre est un silence 
paradoxalement « habité », ou prégnant, c'est-à-dire un silence lourd de bruits muets ? Et surtout, comment rendre le silence par le truchement des mots, qui sont tout sauf silencieux ? Bref, comment rendre le silence autrement que par le silence précisément?

9 La réponse à ce paradoxe pourrait bien être la couleur elle-même. La couleur semble en effet avoir cette faculté d'être expressive tout en restant fondamentalement silencieuse, comme le rappelle le titre du livre de Jacqueline Lichtenstein, La Couleur éloquente ${ }^{18}$. Si la couleur est bel et bien le langage silencieux mais éloquent de la peinture, c'est-à-dire une forme d'expression silencieuse de même qu'une forme d'expression du silence, alors peut-être pourrait-elle rendre, par analogie, le silence « habité » à la fois du tableau de Matisse et de la maison présentés dans la nouvelle. L'expression quelque peu incongrue " silence habité ", à laquelle fait écho plus tôt dans la nouvelle l'expression oxymoronique similaire « le vacarme sourd d'une forme de silence » («the background din of some sort of silence ", 32), fait référence à ce que nous pourrions plus communément appeler un "bruit blanc", l'accumulation de bruits créés par des objets familiers tels que les télévisions, les machines à laver, les sèche-linge, les stéréos, etc, dont la présence bruyante, si l'on n'y prête pas attention, cesse d'être perçue comme telle au bout d'un moment. Dans le cas de notre nouvelle, qui s'ouvre par une visite guidée, effectuée par le narrateur, des trois étages de la maison à travers les différents bruits qui émanent de chaque pièce, c'est exactement ce que nous avons : une maison peuplée de bruits divers qui, réunis, s'annulent et deviennent précisément «le vacarme sourd d'une forme de silence », un «bruit blanc » en d'autres termes. Or, si nous allons plus loin, le choix lexical de «blanc» dans cette expression est intéressant en soi en ce sens que le blanc est aussi, dans le domaine des couleurs de la lumière, la réunion de toutes les couleurs, tout comme un "bruit blanc", en étant la synthèse de toutes les fréquences à l'intérieur d'un intervalle donné, est la réunion de tous les bruits à l'intérieur du domaine des sons. Par analogie, nous pourrions dire que si les «bruits blancs » sont l'équivalent du silence de tous les sons, alors la couleur blanche est l'équivalent du silence de toutes les couleurs, en d'autres termes l'expression suprême du silence inhérent des couleurs. Si tel est le cas, une page "blanche", comme celle figurée dans le tableau Le Silence habité des maisons, semblerait alors être le meilleur vecteur pour essayer de rendre textuellement le silence même de ce tableau. Le tableau pourrait alors se lire comme contenant paradoxalement son propre rendu textuel. Toutefois, une page blanche à l'écrit est aussi une métaphore de la panne d'inspiration du romancier, cette paralysie qui l'empêche de s'exprimer par des mots, et à ce titre la page blanche apparaît comme une bonne façon d'exprimer les limites $\mathrm{du}$ langage. Ce que nous avons par conséquent dans cette nouvelle, c'est l'accomplissement textuel d'une sorte d'impossibilité fondamentale, l'impossibilité d'exprimer ou de transcrire, en mots ou par un autre medium, le silence inhérent à la peinture doublement évoqué ici dans un tableau ayant précisément pour sujet le silence. Plus qu'une simple expérimentation en intermédialité verbale et visuelle, la nouvelle "Art Work » semble ainsi poser directement le problème des limites de la verbalisation des images, et également, à sa suite, le problème des limites de la visualisation des textes, récemment théorisés par des critiques poststructuralistes tels que James Elkins dans son livre On Pictures and the Words that Fail Them ${ }^{19}$. Ce que Elkins et d'autres encore soulignent, c'est l'irréductibilité fondamentale des images et de leurs composantes, telles que les couleurs, liées selon Elkins à la notion de " trace " picturale. Tout comme Robin qui essaie en vain d'exprimer dans ses tableaux la "terreur infinie de l'éclat des couleurs" (the « infinite terror of the brilliance of color », 72), Byatt semble essayer en vain d'exprimer dans ses textes la «terreur infinie du silence des couleurs», et peut-être plus 
généralement la "terreur infinie du silence des images ». Son entreprise littéraire est par conséquent une entreprise qui contient ses propres limites, peut-être même son propre échec, puisque Byatt, telle Pénélope, est à jamais condamnée à tisser et défaire en même temps la trame de son récit dans sa tentative impossible de rendre par des mots l'univers des images. À ce titre, sa nouvelle se présente comme davantage qu'un simple hommage à Matisse ou à la Couleur matissienne, doublé d'une expérimentation esthétique par le biais de la couleur. Tout comme Robin dans ses tableaux, c'est plus généralement le problème de la représentation et de ses limites que Byatt pose ici :

But Debbie saw that it [Robin's painting] was a serious attempt at a serious and terrible problem, an attempt to answer the question every artist must ask him or herself, at some time, why bother, why make representations of anything at all?

Ce qui débute comme une tentative pour rendre le silence, le silence d'un tableau en particulier et le silence de la peinture en général, débouche, à travers un hommage à la couleur et à sa propre forme de silence, sur un questionnement de l'impossibilité fondamentale de toute représentation. À ce constat d'impossibilité s'ajoute un constat d'ineffabilité. En effet, de même que tout le monde disait de Robin, quand il était jeune et qu'il peignait « de petites choses aux couleurs éclatantes dans de vastes aplats de gris, de beige sombre et de beige clair » («small bright things in large expanses of grey and buff and beige »), « il a vraiment quelque chose »; « il a quelque chose » ( He's got something " or « He's got something», 56), le seul mot que Debbie et sa nouvelle femme de ménage, Mrs Stimpson, parviennent à prononcer à la toute fin de la nouvelle devant le tableau de Robin et ses couleurs fraîchement restituées, c'est le même "quelque chose " ("something») dénué de sens dans l'expression exactement semblable "It's got something 》 (90). En révélant l'échec des mots devant l'image, la fiction de Byatt, bien qu'elle-même faite de mots, pourrait bien être exactement cela, un hommage paradoxalement silencieux à l'image, en d'autres termes un hommage silencieux au silence même, à travers précisément ce que l'on pourrait peut-être définir comme une écriture du silence. Et pourtant, malgré le caractère impossible de cette entreprise, il est indéniable que la fiction de Byatt, telle qu'elle est, a bel et bien elle aussi «quelque chose ".

\section{BIBLIOGRAPHIE}

Aragon, L. Matisse-en-France. Paris : Fabiani, 1943.

Byatt, A.S. Portraits in Fiction. London: Chatto and Windus, 2001.

Byatt, A.S. The Matisse Stories. London: Chatto and Windus, 1993.

Cousseau, H.-C. (ed.). Colour since Matisse. New York: Taplinger, 1985.

Elkins, J. On Pictures and the Words that Fail Them. Cambridge: CUP, 1998.

Gowing, L. Matisse. Oxford: OUP, 1979. 
Grimal, P. Dictionnaire de la mythologie grecque et romaine, Paris : PUF, 1951.

Guillerm, J.-P. Des Mots et des couleurs. Lille : PU de Lille, 1986.

Lichtenstein, J. La Couleur éloquente. Paris : Flammarion, 1989.

Mari, C. « De tableau en histoire, d'histoire en tableau : le lecteur-spectateur dans The Matisse Stories de A.S. Byatt ». Études Britanniques Contemporaines 12 (1997). Montpellier : Université Paul Valéry-Montpellier 3.

Matisse, H. Le Silence habité des maisons (1947), huile sur toile, $61 \times 50 \mathrm{~cm}$, coll. part.

Matisse, H. L'Artiste et le modèle reflétés dans le miroir (1937), Black-and-white line drawing, succession Henri Matisse, Paris.

Matisse H. Antoinette au chapeau à plumes, robe juive à mi-corps (1919), crayon, 49x37cm, The Baltimore Museum of Art.

Matisse, H. Femme au chapeau (Madame Matisse) (1905), huile sur toile, 81x65cm, coll. part.

Néret, G. Henri Matisse. Köln : Taschen, 1999.

Shaw, V. The Short Story: A Critical Introduction. London \& New York: Longman, 1983.

\section{NOTES}

1. A.S. Byatt, The Matisse Stories, London: Chatto and Windus, 1993.

2. Catherine Mari, « De tableau en histoire, d'histoire en tableau : le lecteur-spectateur dans The Matisse Stories de A.S. Byatt », Études britanniques contemporaines 12 (Décembre 1997) : 31.

3. Review of Hawthorne's Twice-Told Tales, The Complete Works of Edgar Allan Poe, James A. Harrison (ed.), 17 vols, New York, 1902 ; repr. 1965, XI, 107.

4. Voir à ce sujet Valerie Shaw, The Short Story: A Critical Introduction, London \& New York: Longman, 1983.

5. A.S. Byatt, Portraits in Fiction, London: Chatto and Windus, 2001.

6. Louis Aragon, Matisse-en-France, Paris : Fabiani, 1943.

7. Pour plus de précisions sur le concept d'ekphrasis, voir l'excellent ouvrage de James A.W. Heffernan, Museum of Words: The Poetics of Ekphrasis from Homer to Ashbery, Chicago: University of Chicago Press, 1993.

8. Henri Matisse, Le Silence habité des maisons (1947), huile sur toile, 61 x $50 \mathrm{~cm}$, coll. part.

9. Henri Matisse, L'Artiste et le modèle reflétés dans le miroir (1937). Black-and-white line drawing, succession Henri Matisse, Paris.

10. Lawrence Gowing, Matisse, Oxford: OUP, 1979.

11. Catherine Mari, « De tableau en histoire, d'histoire en tableau : le lecteur-spectateur dans The Matisse Stories de A.S. Byatt », Études britanniques contemporaines 12 (Décembre 1997) : 37.

12. Henri Matisse, Femme au chapeau (Madame Matisse) (1905), huile sur toile, 81 x $65 \mathrm{~cm}$, coll. part.

13. Henri Matisse, Antoinette au chapeau à plumes, robe juive à mi-corps (1919), crayon, $49 \times 37 \mathrm{~cm}$, The Baltimore Museum of Art.

14. Voir à ce sujet Gilbert Néret, Henri Matisse, Köln : Taschen, 1999, 40, 105 \& 187.

15. Voir à ce sujet Pierre Grimal, "Arachné ", Dictionnaire de la mythologie grecque et romaine, Paris : PUF, 1951, 43.

16. Henry-Claude Cousseau (ed.), Colour since Matisse, New York: Taplinger, 1985, 29.

17. Jean-Pierre Guillerm, Des Mots et des couleurs, Lille : Presses Universitaires de Lille, 1986, 13.

18. Jacqueline Lichtenstein, La Couleur éloquente, Paris : Flammarion, 1989.

19. James Elkins, On Pictures and the Words that Fail Them, Cambridge: CUP, 1998. 
INDEX

oeuvrecitee Matisse Stories (The), Art Work 